\title{
Análisis de fase en estudios Gated-SPECT como predictor de mortalidad en pacientes con enfermedad coronaria y función ventricular izquierda deprimida
}

\author{
Francisco Javier García-Gómez ${ }^{\mathrm{a}, *}$, Florencio Javier Luis-Simón ${ }^{\mathrm{b}}$, \\ Agustín Guisado-Rasco ${ }^{\mathrm{y}}$ y Joaquín Víctor Ruíz Franco-Baux ${ }^{\mathrm{a}}$
}

\author{
a Servicio de Medicina Nuclear, Hospital Universitario Virgen del Rocío. Avda. Manuel Siurot s/n, 41013, Sevilla, España \\ b Servicio de Radiofísica Hospitalaria, Hospital Universitario Virgen del Rocío. Avda. Manuel Siurot s/n, 41013, Sevilla, España \\ c Servicio de Cardiología, Hospital Universitario Virgen del Rocío. Avda. Manuel Siurot s/n, 41013, Sevilla, España
}

Recibido el 5 de mayo de 2017; aceptado el 4 de octubre de 2017

Disponible en Internet el 12 de abril de 2018

\section{PALABRAS CLAVE \\ Gated-SPECT; \\ Análisis de fase; \\ Ancho de banda; \\ Predictor de muerte; \\ Pronóstico}

\begin{abstract}
Resumen
Antecedentes: La enfermedad coronaria es una de las principales causas de morbimortalidad en los países occidentales. En etapas avanzadas de la enfermedad, los procesos de remodelación miocárdica pueden conducir a insuficiencia cardíaca progresiva y disfunción ventricular izquierda. El análisis de fase de los estudios de perfusión miocárdica Gated-SPECT muestra parámetros que han sido caracterizados como marcadores válidos de asincronía ventricular. Objetivo: Evaluar los parámetros del análisis de fase en Gated-SPECT como predictores independientes de mortalidad en pacientes con enfermedad coronaria avanzada e insuficiencia ventricular izquierda.

Materiales y método: Estudio retrospectivo de cohortes históricas de 185 pacientes consecutivos (140 hombres; edad media=67,6 $\pm 12,7$ años) a los que, entre enero de 2009 y marzo de 2011, se les hizo estudio isotópico de perfusión miocárdica con estimulación farmacológica con resultado positivo para isquemia/necrosis con $\mathrm{FEVI} \leq 55 \%$. Adicionalmente, se les realizó seguimiento medio de $32,4 \pm 10,5$ meses registrándose la aparición de eventos cardíacos mayores (infarto agudo de miocardio no mortal, ingreso hospitalario y revascularización coronaria tardía) y mortalidad total.

Resultados: Durante el seguimiento se registraron eventos mayores en 51 pacientes así como 28 fallecimientos, de los cuales, $82,1 \%$ mostró valores alterados de los parámetros de fase: media $=141,1^{\circ} \pm 17,6^{\circ}$; desviación estándar $=15,8^{\circ} \pm 10,1^{\circ}$; ancho de banda $=59,1^{\circ} \pm 36^{\circ}$ y $\mathrm{FEVI}=42,4 \% \pm 10,8 \%$. El análisis de Cox mostró al ancho de banda como un predictor independiente de muerte, disminuyendo significativamente la supervivencia y aumentando el riesgo de muerte (hazard ratio $=2,68 ; \mathrm{p}<0,05$ ).
\end{abstract}

\footnotetext{
* Autor para correspondencia.

Correo electrónico: javier191185@gmail.com (F.J. García-Gómez).
} 


\section{KEYWORDS}

Gated-SPECT;

Phase analysis; Bandwidth; Predictor of death; Prognosis

Conclusiones: El ancho de banda en el análisis de fase se comporta como un predictor independiente de muerte en pacientes con miocardiopatía conocida y FEVI deprimida.

( $) 2018$ Sociedad Colombiana de Cardiología y Cirugía Cardiovascular. Publicado por Elsevier España, S.L.U. Este es un artículo Open Access bajo la licencia CC BY-NC-ND (http:// creativecommons.org/licenses/by-nc-nd/4.0/).

\title{
Phase analysis in Gated-SPECT studies as a predictor of mortality in patients with coronary diseases and impaired left ventricular function
}

\begin{abstract}
Background: Coronary disease is one of the main causes of morbidity and mortality in western countries. In the advanced stages of the disease the myocardial remodelling processes can lead to progressive heart failure and left ventricular impairment. The phase analysis of Gated-SPECT studies of myocardial perfusion show parameters that have been characterised as valid marker of ventricular asynchrony.

Objective: To evaluate the phase analysis parameters in Gated SPECT as independent predictors of mortality in patients with advanced coronary disease and left ventricular failure.

Materials and method: A retrospective historic cohort study was conducted on 185 consecutive patients (140 males; mean age $=67.6 \pm 12.7$ years) on whom, between January 2009 and March 2011, an isotope myocardial perfusion study was carried out with pharmacologic stimulation and with a positive result for ischaemia / necrosis, and with a LVEF $\leq 55 \%$. A mean follow-up of $32.4 \pm 10.5$ months was also performed, recording the appearance of major cardiac events (nonfatal acute myocardial infarctions, hospital admission, delayed coronary revascularisation, and total mortality.

Results: Major events were recorded in 51 patients during follow-up. There were also 28 deaths, of which $82.1 \%$ showed abnormal values of the phase parameters: media $=141.1^{\circ} \pm 17.6^{\circ}$; standard deviation $=15.8^{\circ} \pm 10.1^{\circ}$; bandwidth $=59.1^{\circ} \pm 36^{\circ}$, and LVEF $=42.4 \% \pm 10.8 \%$. The Cox analysis showed the bandwidth as an independent predictor of death, significantly reducing the survival and increasing the risk of death (hazard ratio=2.68; $P<.05$ ).

Conclusions: The bandwidth in the phase analysis behaves as an independent predictive factor in patients with known myocardial disease and an impaired LVEF.

(c) 2018 Sociedad Colombiana de Cardiología y Cirugía Cardiovascular. Published by Elsevier España, S.L.U. This is an open access article under the CC BY-NC-ND license (http:// creativecommons.org/licenses/by-nc-nd/4.0/).
\end{abstract}

\section{Introducción}

La enfermedad coronaria es una de las principales causas de morbilidad y mortalidad en los países occidentales. En etapas avanzadas de la enfermedad, los procesos de remodelación del miocardio pueden conducir a insuficiencia cardíaca progresiva y disfunción ventricular izquierda ${ }^{1}$.

El estudio de perfusión miocárdica con tomografía computadorizada por emisión de fotón único sincronizada (Gated-SPECT), permite el análisis de fase del movimiento sistólico del ventrículo izquierdo basado en el histograma de la función de Fourier, en la que se consiguen caracterizar parámetros automatizados, objetivos, reproducibles y válidos, útiles para valorar el sincronismo mecánico del ventrículo izquierdo y no sólo el sincronismo eléctrico ${ }^{2}$. Una de las variables es el ancho de banda del histograma que incluye al $95 \%$ de esa distribución medida en grados $\left({ }^{\circ}\right)$. Otra forma de medir la dispersión de la contracción es mediante el cálculo de la desviación estándar de la media y la entropía, que se expresa de $0 \%$ a $100 \%$. La sincronía perfecta correspondería al 0\% de entropía, considerándose alterada de forma significativa a partir del $60 \%$ y correspondiendo al $100 \%$ la asincronía máxima teórica ${ }^{3}$.

La asincronía ventricular izquierda tiene importantes aplicaciones clínicas como predictor de la respuesta a la terapia de resincronización cardíaca ${ }^{4}$. Además, podría ser también un factor de riesgo de eventos cardíacos (infarto agudo de miocardio, hospitalización por causa cardíaca o necesidad de revascularización tardía) durante el seguimiento a largo plazo en una población de pacientes con sospecha o enfermedad coronaria conocida ${ }^{5,6}$. Su cálculo en el estudio de perfusión isotópica permite evaluar en un único estudio la existencia de patología isquémica miocárdica, calcular parámetros funcionales del ventrículo izquierdo, determinar la viabilidad de la pared miocárdica y valorar el sincronismo ventricular.

El objetivo de este trabajo fue evaluar la asincronía ventricular izquierda, caracterizada mediante el análisis de fase de la sístole del ventrículo izquierdo en estudios GatedSPECT de perfusión miocárdica con 99mTc-tetrofosmina, como predictor de muerte por causa cardíaca o de cualquier causa, en pacientes con enfermedad coronaria avanzada y fracción de eyección del ventrículo izquierdo (FEVI) 
disminuida, así como predictor de mal pronóstico con base en la aparición de eventos cardíacos menores durante el seguimiento.

\section{Material y métodos}

\section{Diseño y sujetos}

Se llevó a cabo un estudio retrospectivo de cohortes históricas entre enero de 2009 y marzo de 2011, de pacientes consecutivos remitidos al servicio de medicina nuclear para estudio de perfusión miocárdica por sospecha de enfermedad coronaria. Se incluyeron los pacientes que mostraron signos de isquemia o necrosis miocárdica en el estudio isotópico de perfusión y con fracción de eyección del ventrículo izquierdo menor o igual al 55\%.

Se excluyeron aquellos con antecedentes personales de arritmia maligna, miocardiopatía dilatada, alteraciones de la conducción (bloqueo completo de la rama izquierda del Haz de His, bloqueo completo de la rama derecha del Haz de His, bloqueo de rama derecha con hemibloqueo anterior de la rama izquierda, fibrilación auricular), marcapasos implantado o terapia de resincronización previa, con el fin de evitar artefactos en la medición de los parámetros de fase ${ }^{7}$. Se excluyeron de la muestra también aquellos pacientes con historia de revascularización dentro de los primeros 90 días tras la realización del estudio de perfusión miocárdica.

La población final fue de 185 pacientes (140 hombres/45 mujeres), con una edad media de $67,6 \pm 12,7$ años y un seguimiento medio de $32,4 \pm 10,5$ meses.

\section{Estudio de perfusión miocárdica}

A todos los pacientes se les realizó un estudio de perfusión miocárdica tras estrés farmacológico con adenosina ( $160 \mathrm{mcg} / \mathrm{kg}$ de peso/minuto durante 6 minutos) en protocolo de un día luego de la administración de una actividad de $370 \mathrm{MBq}$ de $99 \mathrm{mTc}$-tetrofosmina en la fase de estrés y de $888 \mathrm{MBq}$ en la de reposo adquirida en modo Gated-SPECT. Las imágenes se adquirieron en una gammacámara Siemens E.CAM (Siemens Medical Solutions, Erlangen, Alemania) de doble cabezal, posicionados a $90^{\circ}$ con colimador de baja energía y alta resolución, y realizando una órbita no circular con pre-exploración iniciada en oblicua anterior derecha a $45^{\circ}$ con detecciones cada $3^{\circ}$ hasta completar un giro de $180^{\circ}$ en oblicua posterior izquierda y adquisición de 8 frames por ciclo electrocardiográfico en el estudio de reposo GatedSPECT. Las imágenes se analizaron mediante el software QPS $^{\circledR}$ (Quantitative Perfusion SPECT) y QGS $^{\circledR}$ Quantitative Gated SPECT) (Cedars-Sinai Medical Center, Los Ángeles, CA).

Los límites de normalidad en los parámetros de fase se establecieron de acuerdo con el trabajo de Hamalainen et al. ${ }^{8}$, con un valor de pico de $142,3^{\circ} \pm 13,6^{\circ}$, desviación estándar de $10,2^{\circ} \pm 6,1^{\circ}$ y ancho de banda de $30,9^{\circ} \pm 12,7^{\circ}$.

\section{Variables}

Se registraron variables sociodemográficas (edad, sexo, fecha del estudio de perfusión miocárdica), así como factores de riesgo cardiovascular (hipertensión arterial, diabetes mellitus, dislipidemia y hábito tabáquico).

Se registraron, además, los parámetros cualitativos del estudio de perfusión miocárdica Gated-SPECT (isquemia miocárdica de grado severo, afectación del territorio de la coronaria descendente anterior y necrosis miocárdica), así como parámetros cuantitativos: volumen telesistólico (VTS), volumen telediastólico (VTD) y FEVI, medidos en mililitros y porcentaje, respectivamente. Para la FEVI se dicotomizaron los valores en dos grupos: $\mathrm{FEVI} \leq 55 \%$ y $\mathrm{FEVI} \leq 35 \%$.

Respecto al análisis de fase de la sístole del ventrículo izquierdo, se recogieron el ancho de banda, el pico y la desviación estándar de la fase, medidos en grados.

Se revisó exhaustivamente la historia clínica digital de los pacientes, registrándose la presencia de eventos cardíacos durante el seguimiento. Se entendieron como eventos cardíacos mayores la muerte de causa cardíaca o cualquier otra causa, y como eventos cardíacos menores los ingresos hospitalarios por insuficiencia cardíaca, el infarto agudo de miocardio no fatal o la necesidad de revascularización tardía (más de 90 días tras el estudio de perfusión miocárdica).

\section{Análisis estadístico}

La información obtenida de cada paciente a lo largo del seguimiento del estudio, se recogió en una base de datos específica, en la que se incluyeron como campos variables sociodemográficas, clínicas al diagnóstico y relacionadas con la técnica y el seguimiento. Posteriormente, estos datos fueron capturados con el paquete estadístico IBM SPSS 21 (SPSS Inc., Chicago, IL, USA) para llevar a cabo el análisis estadístico. Se realizó la depuración de los datos y un análisis descriptivo, mediante técnicas de exploración y de análisis de frecuencias.

Se hizo un análisis exploratorio y descriptivo de los datos. Las variables cuantitativas se expresaron como media \pm desviación estándar (aquellas que no siguieran una distribución normal, se expresaron en mediana y rango intercuartílico) y las variables categóricas como porcentajes. Los datos base se compararon por Chi cuadrado de Pearson para los categóricos y T de student no pareada para los cuantitativos. A continuación, se realizó la curva de supervivencia de Kaplan-Meier incluyendo el test log-rank para cada subgrupo de pacientes, así como el análisis de regresión uniy multivariante de Cox para identificar los factores que de forma independiente predecían dicho remodelado ventricular izquierdo. El impacto para cada variable se expresó como hazard ratio con un intervalo de confianza del $95 \%$. Todos los cálculos se acompañaron de la estimación del riesgo relativo y del intervalo de confianza del 95\% (IC 95\%); se consideró el dintel de significación estadística cuando $p<0,05$.

\section{Resultados}

Al evaluar la población global a estudio se observaron signos sugestivos de isquemia miocárdica de grado severo en 39 pacientes $(21,08 \%)$, afectación del territorio de la arteria coronaria descendente anterior en 104 pacientes $(56,21 \%)$ y signos de necrosis miocárdica en 110 pacientes $(59,45 \%)$. Distribuidos según la localización de las lesiones con defecto de perfusión en las caras miocárdicas, se identificaron 
Tabla 1 Valores recogidos en la población a estudio $(n=185)$, con eventos cardíacos mayores y menores $(n=51)$ y fallecidos $(\mathrm{n}=28)$ para variables sociodemográficas, factores de riesgo cardiovascular, parámetros cualitativos, cuantitativos y del análisis de fase del estudio Gated-SPECT

\begin{tabular}{|c|c|c|c|}
\hline & $\begin{array}{l}\text { Libre de eventos } \\
(\mathrm{n}=134 ; 72,43 \%)\end{array}$ & $\begin{array}{l}\text { Eventos cardíacos } \\
(n=51 ; 27,57 \%)\end{array}$ & $\begin{array}{l}\text { Muerte }(n=28 ; \\
15,14 \%)\end{array}$ \\
\hline \multicolumn{4}{|l|}{ Características sociodemográficas } \\
\hline Género masculino, n (\%) & $85(60,71 \%)$ & $36(25,71 \%)$ & $19(13,57 \%)$ \\
\hline Edad (años \pm desviación estándar) & $67,7 \pm 12,6$ años & $66,9 \pm 13,2$ años & $73,0 \pm 12,4$ años \\
\hline \multicolumn{4}{|l|}{ Factores de riesgo cardiovascular } \\
\hline Hipertensión arterial & $119(88,80 \%)$ & $45(88,23 \%)$ & $25(89,28 \%)$ \\
\hline Diabetes mellitus & $102(76,11 \%)$ & $38(74,50 \%)$ & $20(71,42 \%)$ \\
\hline Dislipidemia & $113(84,32 \%)$ & $41(80,39 \%)$ & $23(82,14 \%)$ \\
\hline \multicolumn{4}{|l|}{ Parámetros cualitativos Gated-SPECT } \\
\hline Afectación territorio CDA & $72(53,73 \%)$ & $32(62,74 \%)$ & $19(67,85 \%)$ \\
\hline Isquemia severa & $29(21,64 \%)$ & $10(19,60 \%)$ & $5(17,85 \%)$ \\
\hline Necrosis & $80(59,70 \%)$ & $30(58,82 \%)$ & $18(64,28 \%)$ \\
\hline \multicolumn{4}{|l|}{ Parámetros cuantitativos Gated-SPECT } \\
\hline VTS & $60,81 \pm 21,95 \mathrm{ml}$ & $62,35 \pm 22,51 \mathrm{ml}$ & $70,75 \pm 28,00 \mathrm{ml}$ \\
\hline VTD & $112,8 \pm 29,31 \mathrm{ml}$ & $114,5 \pm 29,29 \mathrm{ml}$ & $119,80 \pm 31,26 \mathrm{ml}$ \\
\hline FEVI & $46,96 \pm 7,81 \%$ & $46,64 \pm 7,83 \%$ & $42,42 \pm 10,79 \%$ \\
\hline \multicolumn{4}{|l|}{ Parámetros del análisis de fase } \\
\hline Pico & $52,26 \pm 29,63^{\circ}$ & $52,23^{\circ} \pm 30,57^{\circ}$ & $59,14 \pm 36,06^{\circ}$ \\
\hline Ancho de banda & $136,87 \pm 23,73^{\circ}$ & $134,00^{\circ} \pm 18,27^{\circ}$ & $141,15 \pm 17,58^{\circ}$ \\
\hline Desviación estándar & $14,90 \pm 9,32^{\circ}$ & $14,66^{\circ} \pm 9,35^{\circ}$ & $15,78 \pm 10,07^{\circ}$ \\
\hline
\end{tabular}

CDA: arteria coronaria descendente anterior, VTS: volumen telesistólico, VTD: volumen telediastólico, FEVI: fracción de eyección del ventrículo izquierdo.

92 lesiones en la cara anterior, 29 lesiones en la cara septal, 123 lesiones en la cara inferior, 42 lesiones en la cara lateral y 28 lesiones en el ápex verdadero. Respecto a la FEVI, 16 pacientes presentaban una fracción de eyección $\leq 35 \%$ $(8,64 \%)$, siendo entre $36 \%$ y $55 \%$ en el resto de pacientes de la muestra.

En cuanto a la valoración de los parámetros del análisis de fase, se obtuvieron unos valores medios: pico $=136^{\circ} \pm 22,3$; desviación estándar $=14,8^{\circ} \pm 9,3$; ancho de banda $=52,3^{\circ} \pm 29,8$; y $\mathrm{FEVI}=49,9 \pm 7,8$ para la población total a estudio. Estos datos se resumen en la tabla 1.

Durante el tipo de seguimiento se registraron eventos mayores en 51 pacientes, con una prevalencia de 20 casos de infartos agudos de miocardio, 26 ingresos hospitalarios por insuficiencia cardíaca y 30 revascularizaciones tardías. En cuanto a los decesos, 28 pacientes fallecieron por causa cardíaca/cualquier otra causa durante el periodo de seguimiento. De los 28 pacientes que fallecieron, $23(82,1 \%)$ presentaron valores del ancho de banda por encima del rango de normalidad para su sexo, con una significación estadística de $\mathrm{p}=0,033$ (test de Chi cuadrado de Pearson) y de $p=0,034$ (estadístico exacto de Fisher). En la figura 1 se muestra el interfaz característico del análisis de fase en un paciente con alteraciones en los parámetros de sincronía de la contractilidad ventricular, pudiéndose observar defectos en la perfusión miocárdica, al igual que de los parámetros cuantitativos, cualitativos y cuantitativos tanto del diagrama del análisis de fase como en el mapa polar.

Se observó, además, una correlación inversa alta entre el valor de FEVI y el VTS (R:-0,787, $p<0,001)$, una correla- ción inversa moderada entre la FEVI y desviación estándar (R:-0,441, $p<0,001)$ y de la FEVI con el ancho de banda (R:-0,448, $p<0,001)$, una correlación directa moderada entre el VTS y la desviación estándar (R:0,467, $\mathrm{p}<0,001)$ y entre el VTS y el ancho de banda (R:0,494, $p<0,001)$, así como una correlación directa moderada entre el VTD y la desviación estándar $(\mathrm{R}: 0,378, \mathrm{p}<0,001)$ y del VTD con el ancho de banda (R:0,387 $p<0,001)$.

Finalmente, el modelo de regresión de Cox muestra el ancho de banda como predictor independiente de muerte, disminuyendo significativamente la supervivencia en la curva de Kaplan-Meier (fig. 2) y aumentando el riesgo de muerte un $268 \%$ respecto a quienes no presentan alteración en el ancho de banda (hazard ratio: 2,68; $\mathrm{p}<0,05)$. Otros parámetros como la afectación del territorio de la coronaria descendente anterior y la presencia de necrosis miocárdica se asociaron con mayor tendencia a mortalidad aunque no alcanzaron valores de significación estadística (hazard ratio: 1,$64 ; p=0,220$ y hazard ratio: 1,$36 ; p=0,441$, respectivamente). El resto de parámetros incluidos en el análisis univariante de Cox se muestran en la tabla 2.

\section{Discusión}

La sincronía de la contracción del ventrículo izquierdo es esencial para el funcionamiento correcto del corazón y es, por tanto, un factor determinante para el rendimiento del mismo. La contracción asincrónica del miocardio se asocia 


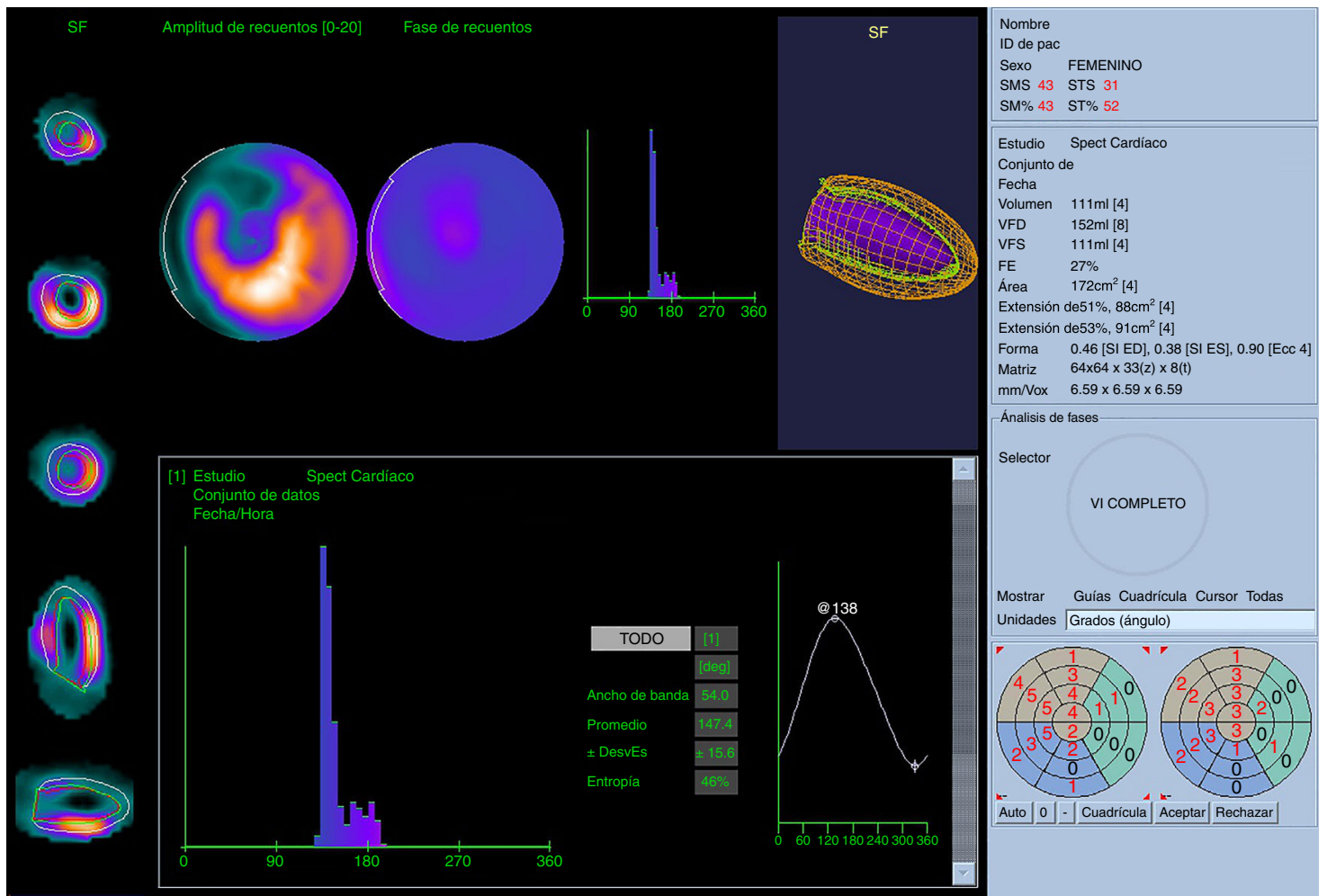

Figura 1 Análisis de fase en paciente mujer de 57 años, con defectos de perfusión en términos de necrosis transmural de la cara anterior y FEVI: $27 \%$ en el estudio de perfusión miocárdica Gated-SPECT. Los parámetros de fase se muestran aumentados respecto a los valores de normalidad, con alteración cualitativa del diagrama y cuantitativa con valores de ancho de banda: $54,0^{\circ}$, pico: $147,4^{\circ}$ y desviación estándar $15,6^{\circ}$.

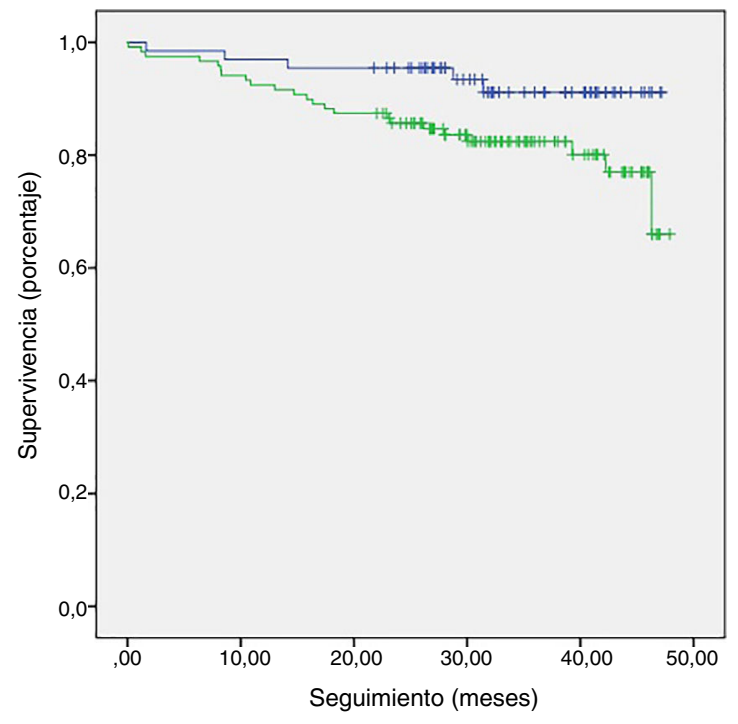

Figura 2 Curva de supervivencia de Kaplan-Meier, en la que se observa una diferencia significativa en la mortalidad en pacientes con alteración del ancho de banda (línea verde) respecto a los pacientes con ancho de banda dentro del rango de normalidad (línea azul), con Log Rank (Mantel-Cox), p=0,037.

con cambios en la perfusión miocárdica y en la remodelación concéntrica del ventrículo izquierdo, lo que conduce a la dilatación ventricular y al deterioro progresivo de la función cardíaca $^{9-11}$.
La asincronía ventricular izquierda, se considera un predictor independiente de mortalidad de cualquier causa en pacientes con miocardiopatía isquémica o sin ella con tiempo de QRS alargado ${ }^{12,13}$, así como predictor de efectos del remodelado, mejoría clínica y supervivencia tras terapia de resincronización ${ }^{4,14}$. Trabajos previos indican cómo la asincronía ventricular izquierda medida con diferentes técnicas de imagen, suponía un peor pronóstico en pacientes con insuficiencia sistólica moderada ${ }^{15}$.

El análisis de fase se basa en el efecto de volumen parcial, de modo que permite obtener una imagen funcional pixel a pixel de la curva de actividad en el pool sanguíneo respecto al tiempo, donde el ángulo de fase de cada pixel, analizado en el borde endocárdico, es proporcional al momento de la máxima reducción de conteos (máxima motilidad ventricular) ${ }^{16}$, en tanto que el recuento máximo regional es proporcional al espesor de la pared miocárdica de la misma región ${ }^{17}$. Es así como el análisis de fase aproxima dicha variación en el espesor de la pared con las funciones armónicas de Fourier para medir el inicio y, por consiguiente, la sincronía de la contracción mecánica.

La asincronía ventricular puede ser valorada mediante diferentes técnicas no invasivas ${ }^{18}$ como la resonancia magnética o la ecocardiografía ${ }^{19}$, si bien la medición de estos parámetros de asincronía mediante Gated-SPECT suponen el empleo de un procedimiento semiautomático objetivo, fiable, reproducible ${ }^{8,20,21}$, que además aporte información sobre la perfusión y la función ventricular izquierda, especialmente la fracción de eyección, los 
Tabla 2 Parámetros predictores de mortalidad incluidos en el análisis univariante de Cox ( $\mathrm{n}=185)$. Significación estadística: $p<0,05$. (FEVI: fracción de eyección del ventrículo izquierdo)

\begin{tabular}{llll}
\hline & Hazard ratio & $95 \%$ IC & Valor de $\mathrm{p}$ \\
\hline Diabetes mellitus & 0,76 & 0,334 a 1,728 & 0,511 \\
Ancho de banda & 2,68 & 1,020 a 7,060 & 0,046 \\
FEVI <35\% & 1,81 & 0,626 a 5,251 & 1,814 \\
Volumen telesistólico $<70 \mathrm{ml}$ & 2,39 & 1,125 a 4,984 & 0,023 \\
Afectación de la arteria descendente anterior & 1,64 & 0,743 a 3,635 & 0,220 \\
Isquemia severa & 0,93 & 0,353 a 2,450 & 0,883 \\
Necrosis miocárdica & 1,36 & 0,623 a 2,959 & 0,441 \\
FEVI medida en Gated-SPECT (\%) & 0,93 & 0,893 a 0,969 & 0,000 \\
\hline
\end{tabular}

volúmenes telesistólico y telediastólico, la motilidad de la pared y el engrosamiento y la cuantificación de los defectos de perfusión ${ }^{1,22}$.

Trabajos recientes han demostrado cómo el análisis de fase es una herramienta útil en la valoración de la asincronía cardíaca secundaria al remodelado de la enfermedad coronaria, así como predictor de eventos cardíacos mayores o de fallecimiento ${ }^{5,6}$, independientemente de otros parámetros como el déficit total de perfusión o una fracción de eyección severamente deprimida ${ }^{1}$.

Además, se trata de una herramienta automatizada, que ha demostrado alta reproducibilidad (escasa variación en la medición por un mismo operador en diferentes momentos o por diferentes operadores), y alta repetitividad (escasa variación entre mediciones sucesivas) ${ }^{23,24}$, siendo esta una fortaleza de la técnica.

En este punto, cabe señalar que los parámetros del análisis de fase de la sístole del ventrículo izquierdo son una variable continua $y$, aunque se correlaciona bien con el aumento de asincronía miocárdica, su uso dicotomizado (parámetros del análisis de fase dentro de la normalidad frente a aumentados) se basa en los valores obtenidos en estudios unicéntricos con pacientes con complejo QRS aumentado ${ }^{4}$. Algunos trabajos fundamentales han propuesto valores de normalidad para tal finalidad: Hamalainen $\mathrm{H}$, et al. ${ }^{8}$ publicó los valores de referencia para los parámetros del análisis de fase mediante Gated-SPECT en estrés y en reposo con el software Quantitative Gated SPECT (CedarsSinai Medical Center, Los Ángeles, California). Por otro lado, los trabajos de Chen et al. ${ }^{24}$ y Romero-Farina et al. ${ }^{25}$, aportan rangos de normalidad para el análisis de fase con el software SyncTool ${ }^{T M}$ (Emory Cardiac Toolbox ${ }^{T M}$, Emory University, Atlanta, Georgia, USA). Debido a la incompatibilidad de valores entre ambos programas de procesamiento, se tomaron como rangos de normalidad los publicados en el trabajo de Hamalainen et al. ${ }^{8}$ para los estudios Gated-SPECT en reposo.

En cuanto a su utilidad clínica, en los últimos años se han publicado trabajos interesantes acerca de su capacidad para predecir la respuesta a la terapia de resincronización 1,4,26-28. En cambio, pocos trabajos han evaluado su utilidad en la predicción de la mortalidad de causa cardíaca u otra causa ${ }^{1,6}$. Según nuestro conocimiento, este trabajo es el estudio prospectivo con mayor tamaño muestral hasta la fecha para determinación de parámetros de fase con QGS ${ }^{\circledR}$ como predictores de muerte.
En otro sentido, la determinación del umbral de normalidad para la FEVI es una cuestión discutida en la actualidad ${ }^{29}$. $\mathrm{Si}$ bien las guías clínicas definen que una FEVI normal o conservada es aquella $>50 \%$, estudios recientes han demostrado que pacientes con FEVI comprendidas en el rango bajo de la normalidad (entre $50 \%$ y $55 \%$ ) tienen mayor riesgo de morbilidad y mortalidad ${ }^{30}$. Por ello, en este trabajo, se consideraron pacientes con FEVI deprimida quienes mostraran valores $\leq 55 \%$. Con el ánimo de valorar posibles diferencias en los parámetros de fase en relación con la severidad de la afectación de la FEVI, los pacientes fueron divididos en dos grupos: FEVI deprimida cuando $\leq 55 \%$ y FEVI severamente deprimida cuando $\leq 35 \%$.

En este trabajo, el $82,1 \%$ de los pacientes que fallecieron durante el periodo de seguimiento, mostraron alteración de los parámetros de fase respecto al umbral de normalidad para su edad, con valores, fundamentalmente el ancho de banda, de $59,12^{\circ} \pm 36$, alcanzando una significación estadística de $\mathrm{p}=0,033$ (test de Chi cuadrado de Pearson) y de $\mathrm{p}=0,034$ (estadístico exacto de Fisher). Estos datos concuerdan con los de Uebleis et al. ${ }^{1}$ sobre 135 pacientes, alcanzando también la significación estadística $(p=0,005)$. Asimismo, del trabajo publicado por Pazhenkottil et al. ${ }^{6}$ se recoge una tasa significativamente más alta de eventos cardíacos mayores en pacientes con alteraciones en la sincronía ventricular $(p<0,001)$. No obstante, el análisis de fase fue realizado en dicho trabajo con Emory Cardiac Toolbox ${ }^{\mathrm{TM}}$, sin que se pudiera establecer una comparación directa de parámetros. Esta alteración en el ancho de banda se traduce en una disminución significativa de la supervivencia, comportándose como factor de riesgo independiente de muerte e incrementando el riesgo un $268 \%$ respecto a quienes no presentan alteración en el ancho de banda (hazard ratio: 2,68 ; IC95\%: 1,020 a 7,060; p=0,04).

Otros parámetros cualitativos del estudio de perfusión miocárdica, como la presencia de necrosis miocárdica y la afectación predominante del territorio de la arteria descendente anterior tienen importancia pronóstica ${ }^{29}$. En nuestro estudio, ambos parámetros se asocian con mayor tendencia a la mortalidad aunque no alcanzaron valores de significación estadística. La afectación predominante del territorio de la arteria descendente anterior también fue considerada en el análisis univariante de Cox en trabajos previos ${ }^{1,6}$, sin que se alcanzase significación estadística en ninguno de ellos. Asimismo, como cabría esperar, se observa mayor tendencia a la mortalidad aunque sin 
significación estadística en pacientes con $\mathrm{FEVI}<35 \%$. Este punto podría estar condicionado por el bajo número de pacientes con esta circunstancia clínica. Por último, tampoco se observaron diferencias estadísticamente significativas en la mortalidad en relación con el resto de variables estudiadas (tabla 2).

Respecto a las curvas de supervivencia, se observó un descenso significativo de la misma en pacientes con alteración del ancho de banda, con un valor de log-rank (Mantel-Cox): $p=0,037$. En los trabajos de Chen et al. ${ }^{24}$ y Romero-Farina et $a .^{25}$, se describe un descenso de la supervivencia en pacientes con alteraciones de la sincronía, sin que se especifique la correlación con un determinado parámetro del análisis de fase, alcanzando en ambos casos un valor de log-rank: $p<0,001$.

\section{Limitaciones}

Conviene considerar aquellos aspectos que puedan suponer una limitación a nuestro trabajo. El primero de ellos es que el análisis de fase sobre el estudio Gated-SPECT se realizó en el estudio en fase de reposo y no en el estudio tras estrés farmacológico, debido a que el protocolo de nuestro centro es de un día y la actividad del radiotrazador administrada en la fase de estrés, resultaría insuficiente para obtener un estudio Gated-SPECT de aceptable calidad. No obstante, se han descrito diferencias menores en los resultados obtenidos del análisis de fase en estudios de perfusión tras estrés farmacológico y en reposo, siendo ambas útiles en el diagnóstico de la asincronía con un coeficiente de correlación entre clases de 0,59 y $0,65^{11}$.

Otro factor a considerar es la adquisición del estudio Gated-SPECT a 8 frames por ciclo electrocardiográfico que, a priori, aportaría menor resolución temporal respecto a una adquisición de 16 frames por ciclo. En este sentido, se ha objetivado una diferencia muy pequeña en los valores del análisis de fase entre ambas técnicas de adquisición, de apenas $0,5^{\circ}$, por lo que no parece ser un factor determinante siempre que los estudios se realicen con un número suficiente de cuentas ${ }^{24}$.

Por último, existe una limitación inherente al carácter retrospectivo de cohortes históricas del trabajo, con una pérdida potencial de datos durante el seguimiento. A este respecto, el tamaño muestral que se presenta, así como la exhaustiva selección de la misma, con exclusión de aquellos pacientes con factores de confusión para la valoración de la asincronía, pretende minimizar este hecho.

\section{Conclusión}

La determinación de los parámetros de asincronía mediante análisis de fase del ventrículo izquierdo en el estudio de perfusión Gated-SPECT, es una técnica semiautomática objetiva, fiable y reproducible, que permite además obtener información sobre la perfusión y la función ventricular izquierda. Particularmente, la alteración del ancho de banda de la fase se comporta como predictor independiente de muerte, con diferencia significativa en la supervivencia y aumento del riesgo de muerte de un $268 \%$, en pacientes con miocardiopatía conocida y FEVI deprimida.

\section{Financiación}

Ninguna.

\section{Conflicto de intereses}

Ninguno.

\section{Bibliografía}

1. Uebleis C, Hellweger S, Laubender RP, Becker A, Sohn HY, Lehner $S$, et al. Left ventricular dyssynchrony assessed by GatedSPECT phase analysis is an independent predictor of death in patients with advanced coronary artery disease and reduced left ventricular function not undergoing cardiac resynchronization therapy. Eur J Nucl Med Mol Imaging. 2012;39:1561-9.

2. Matsuo S. Phase analysis using gated myocardial perfusion single-photon emission computed tomography imaging for evaluating cardiac dyssynchrony. Circ J. 2012;76:1832-3.

3. Gutiérrez L, Peñafort F, Seretti I, Ortego R. Nuevas herramientas diagnósticas en insuficiencia cardíaca: análisis de fase mediante SPECT-Gatillado para la evaluación de sincronía miocárdica. Rev Fed Arg Cardiol. 2013;42:102-12.

4. Henneman MM, Chen J, Dibbets-Schneider P, Stokkel MP, Bleeker GB, Ypenburg C, et al. Can LV dyssynchrony as assessed with phase analysis on gated myocardial perfusion SPECT predict response to CRT? J Nucl Med. 2007;48:1104-11.

5. Goldberg AS, Alraies MC, Cerqueira MD, Jaber WA, Aljaroudi WA. Prognostic value of left ventricular mechanical dyssynchrony by phase analysis in patients with non-ischemic cardiomyopathy with ejection fraction $35-50 \%$ and QRS $<150 \mathrm{~ms}$. J Nucl Med Cardiol. 2014;21:57-66.

6. Pazhenkottil AP, Buechel RR, Husmann L, Nkoulou RN, Wolfrum M, Ghadri JR, et al. Long-term prognostic value of left ventricular dyssynchrony assessment by phase analysis from myocardial perfusion imaging. Heart. 2011;97:33-7.

7. Aguade-Bruix S, Mariscal-Labrador E, Cuberas-Borros G, SabateFernandez M, Pizzi MN, Kisiel-Gonzalez N. MPI phase analysis for assessment of left ventricular contraction dyssynchrony [Abstract]. Eur Heart J. 2011;13 Supplement A. A19.

8. Hamalainen $\mathrm{H}$, Hedman $\mathrm{M}$, Laitinen $\mathrm{T}$, Hedman $\mathrm{A}$, Kivela A, Laitinen T. Reference values for left ventricular systolic synchronyaccording to phase analysis of ECG-gated myocardialperfusion SPECT. Clin Physiol Funct Imaging. 2016 Jul 24, http://dx.doi.org/10.1111/cpf.12379 [Epub ahead of print].

9. Sharma RK, Volpe G, Rosen BD, Donekal S, Fernandes V, Wu $\mathrm{CO}$, et al. Prognostic implications of left ventricular dyssynchrony for major adverse cardiovascular events in asymptomatic women and men: the multi-ethnic study of atherosclerosis. J Am Heart Assoc. 2014;3:1-12.

10. Ovadia M, Abudayyeh I. Risk stratification by quantitation of LV dyssynchrony: a new branch of the field of nuclear cardiology. J Nucl Cardiol. 2014;21:747-52.

11. Tavares A, Peclat T, Lima RS. Prevalence and predictors of left intraventricular dyssynchrony determined by phase analysis in patients undergoing gatedSPECT myocardial perfusion imaging. Int J Cardiovasc Imaging. 2016;32:845-52.

12. Bader H, Garrigue S, Lafitte S, Reuter S, Jais P, Haissaguerre $M$, et al. Intra-left ventricular electromechanical asynchrony. A new independent predictor of severe cardiac events in heart failure patients. J Am Coll Cardiol. 2004;43:248-56.

13. Fauchier L, Marie O, Casset-Senon D, Babuty D, Cosnay P, Fauchier JP. Interventricular and intraventricular dyssynchrony in idiopathic dilated cardiomyopathy: a prognostic study with fourier phase analysis of radionuclide angioscintigraphy. J Am Coll Cardiol. 2002;40:2022-30. 
14. Domenichini G, Burri H, Valzania C, Gavaruzzi G, Fallani F, Biffi $M$, et al. QRS pattern and improvement in right and left ventricular function after cardiac resynchronization therapy: A radionuclide study. BMC Cardiovascular Disorders. 2012;12:27.

15. Marwick TH. Hype and hope in the use of echocardiography for selection for cardiac resynchronization therapy: The Tower of Babel revisited. Circulation. 2008;117:2573-6.

16. Toussaint JF, Peix A, Lavergne T, Kolar P, Alonso C, Froissart $M$, et al. Reproducibility of the ventricular synchronization parameters assessed by multiharmonic phase analysis of radionuclide angiography in the normal heart. Int J Cardiovasc Imaging. 2002;18:187-94.

17. Galt JR, Garcia EV, Robbins WL. Effects of myocardial wall thickness on SPECT quantification. IEEE Trans Med Imaging. 1990;9:144-50.

18. AlJaroudi W, Chen J, Jaber WA, Lloyd SG, Cerqueira MD, Marwick T. Nonechocardiographic imaging in evaluation for cardiac resynchronization therapy. Circ Cardiovasc Imaging. 2011;4:334-43.

19. Sakamaki F, Seo Y, Ishizu T, Yanaka S, Atsumi A, Yamamoto M, et al. Tissue Doppler imaging dyssynchrony parameter derived from the myocardial active wall motion improves prediction of responders for cardiac resynchronization therapy. Circ J. 2012;76:689-97.

20. Trimble MA, Velazquez EJ, Adams GL, Honeycutt EF, PagnaneIli RA, Barnhart HX, et al. Repeatability and reproducibility of phase analysis of gated single-photon emission computed tomography myocardial perfusion imaging used to quantify cardiac dyssynchrony. Nucl Med Commun. 2008;29:374-81.

21. Lin $X, X u H$, Zhao X, Folks RD, Faber TL, Garcia EV, et al. Repeatability of left ventricular dyssynchrony and function parameters in serial gated myocardial perfusion SPECT studies. J Nucl Cardiol. 2010;17:811-6.

22. Berman DS, Germano G. Evaluation of ventricular ejection fraction, wall motion, wall thickening, and other parameters with gated myocardial perfusion single-photon emission computed tomography. J Nucl Cardiol. 1997;4:S169-71.
23. Chung ES, Leon AR, Tavazzi L, Sun JP, Nihoyannopoulos P, Merlino $J$, et al. Results of the Predictors of Response to CRT (PROSPECT) trial. Circulation. 2008;117:2608-16.

24. Chen J, Garcia EV, Folks RD, Cooke CD, Faber TL, Tauxe EL, et al. Onset of left ventricular mechanical contraction as determined by phase analysis of ECG-gated myocardial perfusion SPECT imaging: Development of a diagnostic tool for assessment of cardiac mechanical dyssynchrony. J Nucl Cardiol. 2011;12: 687-95.

25. Romero-Farina G, Aguadé-Bruix S, Candell-Riera J, Pizzi MN, García-Dorado D. Cut-off values of myocardial perfusion gatedSPECT phase analysis parameters of normal subjects, and conduction and mechanical cardiac diseases. J Nucl Cardiol. 2015;22:1247-58.

26. Hou PN, Tsai SC, Lin WY, Cheng CM, Chiang KF, Chang YC, et al. Relationship of quantitative parameters of myocardial perfusion SPECT and ventricular arrhythmia in patients receiving cardiac resynchronization therapy. Ann Nucl Med. 2015;29:772-8.

27. Azizian N, Rastgou F, Ghaedian T, Golabchi A, Bahadorian B, Khanlarzadeh V, et al. LV Dyssynchrony Assessed With Phase Analysis on Gated Myocardial Perfusion SPECT Can Predict Response to CRT in Patients With End-Stage Heart Failure. Res Cardiovasc Med. 2014;3:e20720.

28. Mukherjee A, Patel CD, Naik N, Sharma G, Roy A. Quantitative assessment of cardiac mechanical dyssynchrony and prediction of response to cardiac resynchronization therapy in patients with nonischaemic dilated cardiomyopathy using gated myocardial perfusion SPECT. Nucl Med Commun. 2015;36:494-501.

29. Hobbs FD, Roalfe AK, Davis RC, Davies MK, Hare R. Midlands Research Practices Consortium (MidReC). Prognosis of all-cause heart failure and borderline left ventricular systolic dysfunction: 5 year mortality follow-up of the Echocardiographic Heart of England Screening Study (ECHOES). Eur Heart J. 2007;28:1128-34.

30. Tsao CW, Lyass A, Larson MG, Cheng S, Lam CS, Aragam JR, et al. Prognosis of Adults With Borderline Left Ventricular Ejection Fraction. JACC Heart Fail. 2016;4:502-10. 